\title{
O papel das urolitíases na obstrução uretral em felinos domésticos: Uma revisão de
}

\section{literatura}

\author{
The role of urolithiasis in urethral obstruction in cats: A literature review \\ El papel de la urolitiasis en la obstrucción uretral en gatos: Revisión de la literatura
}

Laura Dias Petricione de Souza

ORCID: https://orcid.org/0000-0003-4731-7577 Universidade Federal de Pelotas, Brasil E-mail:laurapetricionegmail.com Michaela Marques Rocha

ORCID: https://orcid.org/0000-0002-5316-459X Universidade Federal de Pelotas, Brasil E-mail: michaelamr98@ @otmail.com

Juliana Tasende Ferrando

ORCID: https://orcid.org/0000-0002-1737-8523 Universidade Federal de Pelotas, Brasil E-mail: tasendejul@gmail.com

Laura Aparecida Martins de Moraes ORCID: https://orcid.org/0000-0003-3672-8020

Universidade Federal de Pelotas, Brasil

E-mail: laura_m_moraes@outlook.com

Ana Júlia Rodrigues Teixeira Ramos ORCID: https://orcid.org/0000-0002-7522-2703

Universidade Federal de Pelotas, Brasil

E-mail: anajulia.aj@hotmail.com

Adeline Bogo Madril

ORCID: https://orcid.org/0000-0002-0303-5722

Universidade Federal de Pelotas, Brasil

E-mail: adeline_madril@hotmail.com

Maurício Andrade Bilhalva

ORCID: https://orcid.org/0000-0003-2500-0202

Universidade Federal de Pelotas, Brasil

E-mail: mauricioandradebilhalva@gmail.com

Aline do Amaral

ORCID: https://orcid.org/0000-0003-3039-3194

Universidade Federal de Pelotas, Brasil

E-mail: amaralaaline@gmail.com

Rafaela Castanheira Soares

ORCID: https://orcid.org/0000-0001-8157-0133 Universidade Federal de Pelotas, Brasi

E-mail: rafaela.castanheira.soares@gmail.com

Gustavo Antônio Boff

ORCID: https://orcid.org/0000-0002-5837-5302 Universidade Federal de Pelotas, Brasi

E-mail: gustavo_boff@hotmail.com

Júlia Vargas Miranda

ORCID: https://orcid.org/0000-0001-6576-3496 Universidade Federal de Pelotas, Brasil E-mail: juvm@live.com

Vitória Baierle Maggi

ORCID: https://orcid.org/0000-0002-4099-0390 Universidade Federal de Pelotas, Brasil

E-mail: vih_maggi@hotmail.com

Guilherme Albuquerque de Oliveira Cavalcante

ORCID: https://orcid.org/0000-0001-8340-9087 Universidade Federal de Pelotas, Brasil E-mail: guialbuquerque@yahoo.com

Fabiane Borelli Grecco

ORCID: https://orcid.org/0000-0002-3996-315X Universidade Federal de Pelotas, Brasil E-mail: fabianegrecco18@gmail.com 


\title{
Resumo
}

A doença do trato urinário inferior dos felinos (DTUIF) é um problema frequente na rotina clínica, que resulta em alteração no padrão de micção. A obstrução uretral em gatos enquadra-se na DTUIF e representa grande risco à vida, e ocorre principalmente pela formação de urólitos que impedem o fluxo urinário, podendo desencadear uma azotemia pós renal e falência renal aguda obstrutiva. Há diversas hipóteses sobre a formação e desenvolvimento das urolitíases, mas nenhuma delas é muito bem elucidada, podendo ocorrer de forma isolada ou simultânea. Ao longo dos anos, a prevalência do tipo de urólito em felinos foi alterada, principalmente devido a modificação dietética implantada com o objetivo de prevenir o surgimento de urólitos de estruvita, o que aumentou a casuística de oxalato de cálcio em gatos. Esse trabalho tem como objetivo contribuir para o entendimento da fisiopatologia envolvida na formação dos dois principais tipos de urolitíases na espécie felina e auxiliar no diagnóstico, tratamento e prevenção em cada caso.

Palavras-chave: Felinos; Obstrução uretral; Urolitíases.

\begin{abstract}
Feline lower urinary tract disease (FLUTD) is a common problem in clinical routine, resulting in a change in urination pattern. Urethral obstruction in cats fits into FLUDT and represents high risk to life, occuring mainly by the formation of uroliths that stop urinary flow, being able to trigger post renal azotemia and obstructive acute kidney failure. There are many hypothesis about the formation and development of urolithiasis, but none of them are very clear, as it can happen alone or simultaneously. Over the years, the urolith type prevalence in felines has been modified, mainly because of dietary changes implemented to prevent the emergence of struvite uroliths, which increased the cases of calcium oxalate in cats. The current paper has the purpose of contributing to the understanding of the pathophysiology involved in the formation of the main two kinds of urolithiasis in the feline species and assist in the diagnosis, treatment and prevention of each case.
\end{abstract}

Keywords: Cats; Urethral obstruction; Urolithiasis.

\section{Resumen}

La enfermedad del tracto urinario inferior de los felinos (ETUIF) es un problema frecuente en la rutina clínica, que resulta en alteraciones del padrón de micción. La obstrucción uretral en gatos se encuadra en la ETUIF y representa un grande riesgo de vida, ocurriendo principalmente por la formación de urolitos que impiden el flujo urinario, pudiendo desencadenar una azotemia post-renal y el colapso renal obstructivo agudo. Existen diversas hipótesis sobre el suceso y transcurso de las urolitiasis, aunque ninguna de ellas es suficientemente clara, siendo capaz de ocurrir de forma aislada o simultánea. Con el pasar del tiempo, el predominio del tipo de urolitos en felinos fue siendo alterado, principalmente devido a la modificación dietética implantada con el objetivo de evitar la formación de urolitos de estruvita, lo que consecuentemente aumentó la cantidad de urólitos de oxalato de calcio en gatos. Este trabajo tiene como objetivo contribuir para la comprensión de la fisiopatologia implicada el los dos principales tipos de urolitiasis de la especie felina y asistir en su diagnóstico, tratamiento y prevención en cada caso.

Palabras clave: Felinos; Obstrucción uretral; Urolitiasis.

\section{Introdução}

O rim possui diversas funções, entre elas a filtração do sangue para a eliminação de produtos de excreção do metabolismo e a manutenção do equilíbrio hidroeletrolítico (Nelson \& Couto, 2001). Quando há algum tipo de desequilíbrio na homeostase, a azotemia pode ser observada, caracterizada pelo aumento nas concentrações de ureia, creatinina e outras substâncias nitrogenadas não proteicas no sangue, podendo ser de origem pré-renal, renal ou pós-renal (Ettinger \& Feldman, 2004).

A doença do trato urinário inferior dos felinos (DTUIF) é um dos problemas mais frequentes na rotina clínica, sendo observado alterações no padrão de micção dos gatos acometidos (Dulaney, Hopfensperger, Malinowski, Hauptman, \& Kruger, 2017). O perfil dos animais com DTUIF geralmente inclui gatos machos, castrados, sedentários, obesos, entre 1 a 10 anos de idade, domiciliados, que consomem ração seca e bebem pouca água (Martins et al., 2013).

Diferentes tipos de afecções do trato urinário inferior dos felinos apresentam sinais clínicos muito semelhantes, que podem se apresentar isoladamente ou em conjunto (Ferreira, 2013), sendo principalmente hematúria, polaquiúria, estrangúria, micção em local inapropriado, vocalização e a presença ou não da obstrução uretral (Robertson, 2014).

Segundo Cooper (2015), a obstrução uretral é a consequência mais grave da DTUIF, pois pode impedir o fluxo urinário (Lima et al., 2008), desencadeando uma azotemia pós-renal e a falência renal aguda obstrutiva, com risco mediato à vida (Giovaninni \& Piai, 2010). 
Entre as principais causas de obstrução uretral estão as urolitíases, caracterizadas pela presença de urólitos na região do trato urinário (Houston, Vanstone, Moore \& Weese, 2016), podendo ser nefrólitos (rim), ureterólitos (ureter), urocistólitos (bexiga) e uretrólitos (uretra) (Lekcharoensuk et al., 2011).

Assim, o presente trabalho tem como objetivo realizar o levantamento bibliográfico sobre os diferentes tipos de urólitos na espécie felina, para contribuir com o entendimento sobre sua formação, fatores predisponentes, diagnóstico e tratamento.

\section{Metodologia}

O estudo se deu através de uma revisão da literatura entre os meses de janeiro a junho de 2021, utilizando-se das bases de dados PubMed, SciELO, Periódicos Capes e Google Scholar que abordam a temática, com o objetivo de analisar publicações sobre urolítiases, em especial na espécie felina, utilizando-se das palavras chaves: urolítíases, felinos, oxalato de cálcio e estruvita.

O critério de inclusão foi a partir de literatura que tivessem relevância no tema, com conceitos importantes, que abordassem as principais teorias, e autores clássicos que norteiam a literatura do tema abordado, tanto na lingua portuguesa como na inglesa. A exclusão de artigos se baseou naqueles que não atendessem os objetivos propostos nesse trabalho.

\section{Revisão de literatura}

\section{Formação, desenvolvimento e caracterização dos urólitos}

Existem inúmeras teorias a respeito da patogenia dos urólitos. Na teoria da precipitação-cristalização, acredita-se que sua formação se inicia com a presença de cristais de menor solubilidade em uma urina supersaturada, dando início a formação de um núcleo e a manutenção desse urólito (Macphail, 2014). Na teoria núcleo da matriz, acredita-se que uma substância orgânica presente na urina promova a formação inicial do núcleo, podendo ser albumina, globulina ou mucoproteína. Em outra teoria, da inibição da cristalização, considera-se como fator primário a ausência de um inibidor da formação de cristal, que permite o desenvolvimento inicial de um núcleo (Grauer, 2001).

Seu desenvolvimento se dá através de predisposição genética, gênero, defeitos congênitos, desequilíbrio metabólico, alimentação inapropriada, desequilíbrio no pH urinário, baixa ingestão de água, entre outros (Pimenta, Reche-Júnior, Freitas, Kogika \& Hagiwara, 2014; Oyafuso et al., 2010).

Os urólitos mais encontrados nos felinos são a estruvita e o oxalato de cálcio (Little, 2016), sendo os urólitos de urato de amônio, cistina, silicato, xantina, fosfato de cálcio e pirofosfato menos relatados (Assis \& Taffarel, 2018). Sua composição pode ser classificada como simples, quando este tem o predomínio maior ou igual à $70 \%$ de um único mineral, composto, quando apresentam minerais diferentes em camadas distintas e ainda podem ser considerados mistos, apresentando camadas com diferentes tipos de minerais sem predominância de um tipo de mineral (Ulrich, Osborne, Cokley \& Lulich, 2009).

Apesar do aspecto físico da maioria dos cálculos ser considerado um bom indicador da sua composição, é importante salientar que sua composição externa raramente proporciona indicação sobre sua composição interior. Sendo assim, é fundamental a identificação dos tipos de minerais contidos em todas as camadas, principalmente no núcleo, uma vez que dão origem ao cálculo (Moore, 2007).

\section{Principais urólitos em felinos}

Os urólitos compostos por fosfato de amônio magnesiano hexahidratado são conhecidos por urólitos de estruvita. Podem ter formato esférico, elipsoidal ou tetraédrico, se apresentar em diferentes tamanhos e quantidades e na maioria das vezes são formados na bexiga e em urina estéril nos gatos (Guerra, 2018). Porém sua produção pode ser induzida por 
infecções em gatos com menos de um ano e gatos com mais de 10 anos de vida, sendo muito mais raro do que a frequência em cães (Assis \& Taffarel, 2018).

As raças predispostas são o Himalaia, o Persa e o gato doméstico (Stevenson, 2002), com idade média de 5 a 7 anos (Houston, Moore, Favrin \& Hoff, 2003). Nos gatos Rex, Burmês, Abissínio, Azul da Rússia, Sagrado da Birmânia e Siamês, o risco de formação de cálculos de estruvita parece ser mais baixo (Lekcharoensuk, Osborne \& Lulich, 2001).

Os urólitos de estruvita podem ser divididos em três grupos: urólitos de estruvitas estéreis, induzidos por infecção e tampões uretrais de estruvita. Os cálculos estéreis de estruvita são as mais frequentes nos gatos, e se formam sem a existência prévia de uma infecção urinária, estando associado a um conjunto multifatorial. Os urólitos de estruvita estéreis formam-se principalmente em gatos de um a 10 anos e não têm predisposição sexual (Bartges \& Kirk, 2006). Acredita-se que sua formação ocorra pela supersaturação da urina (secundários à baixa ingestão hídrica), por obesidade, sedentarismo, pH alcalino e a alimentação rica em magnésio e fósforo (Filho, Prado, Ribeiro \& Fortes, 2013; Lulich, Osborne, Bartges \& Lekcharoensuk, 2015). Foi demonstrado que em condições onde pH urinário está constantemente elevado, a chance de formação de urólitos de estruvita era duas vezes maior (Grauer, 2015). O aumento do $\mathrm{pH}$ é afetado principalmente pela dieta, mas também pode estar associado à administração de fármacos e desordem tubular renal (Lekcharoensuk, Osborne \& Lulich, 2001).

O segundo mecanismo está associado aos cristais de estruvita induzidos por infecção bacteriana, relacionados à produção de urease. Esse mecanismo é raro em felinos, sendo principalmente observados em gatos com menos de um ano de idade, em gatos idosos com doença renal crônica, animais submetidos a procedimentos como uretrostomia perineal, bem como indivíduos com doenças que predispõem a alterações nas barreiras de defesa contra a infecção do trato urinário (MayerRoenne, Goldstein \& Erb, 2007).

A urease é uma enzima que, em presença de água, hidrolisa a ureia, produzindo moléculas de amônia e dióxido de carbono (Macphail, 2014). A amônia se transforma em amônio, aumenta o pH urinário tornando-o mais alcalino, diminuindo a possibilidade de solubilidade da estruvita. O dióxido de carbono, ao se unir à água, se transforma em ácido carbônico, que ao ser dissociado, produz um aumento de bicarbonato. Nessa urina mais alcalina, ocorre também o aumento da concentração de fosfato (Guerra, 2018).

O terceiro mecanismo está relacionado aos tampões uretrais de estruvita, e é considerado como resultado de uma associação de fatores predisponentes dos cristais de estruvita estéreis e dos induzidos por infecção, sendo esse mecanismo uma das causas mais comuns de obstrução uretral em gatos (Osborne et al., 1995).

Já os urólitos de oxalato de cálcio possuem formato arredondado ou de roseta (Bartge, 2016), são o segundo tipo de cálculos mais relatado em felinos e são frequentemente encontrados na pelve renal e nos ureteres de gatos (Assis \& Taffarel, 2018).

Os fatores de risco associados são gatos com mais de 7 anos de idade (Lekcharoensuk et al., 2000), assim como as raças Persa, Himalaio, Birmaneses, English Shorthair, Exotic Shorthair, Havana Brown, Ragdoll, Foreign Shorthair, Scotish Fold (Little, 2016). Estudos apontam que felinos machos também têm uma maior predisposição em relação à fêmeas (Daudon et al., 2016).

A formação desse tipo de cálculo ocorre quando a urina está supersaturada com cálcio e oxalato (Bartges et al., 1999). Alterações na atividade e quantidade destes íons e a presença de proteínas de elevado peso molecular também pode influenciar na formação destes urólitos (Balaji \& Menon, 1997).

Foi demonstrado que cerca de $35 \%$ dos felinos que apresentaram urolitíase por oxalato de cálcio tinham hipercalcemia idiopática (Midkiff, Chew, Randolph, Center \& DiBartola, 2000), sendo um fator importante no desenvolvimento da doença.

Outras condições como doença renal crônica, acidose metabólica, adenocarcinoma de paratireoide (Osborne, Lulich, Forrester \& Albasan, 2009; Marquez, Klausner \& Osborne, 1995), e hiperparatireoidismo primário e/ou secundário podem 
causar hipercalcemia e hipercalciúria (Grauer, 2015). Deste modo, as concentrações de cálcio ionizado devem ser mensuradas em gatos com a presença de urólitos de oxalato de cálcio para descartar hipercalcemia (Palm \& Westropp, 2011).

Os baixos níveis de fósforo na dieta são um fator de risco na formação de urólitos de oxalato de cálcio em gatos (Lekcharoensuk et al, 2000), pois sua redução está associada à ativação da vitamina D que promove a absorção de cálcio intestinal e hipercalciuria. Dietas formuladas para aumentar a acidez urinária (como as dietas terapêuticas para dissolução de urólitos de estruvita) também podem elevar os riscos de formação de cálculos de oxalato, assim como dietas com baixos teores de sódio e potássio (Bartges \& Kirk, 2006).

Durante anos, a estruvita foi um dos minerais mais comuns na composição dos urólitos e plugs de muco observados em felinos, seguido por oxalato de cálcio (Little, 2012), mas ao longo dos anos ocorreu uma mudança nos tipos de urólitiases, principalmente devido a acidificação das dietas comerciais desenvolvidas para diminuir a precipitação de estruvita, o que ocasionou a maior precipitação de cristais de oxalato de cálcio (Houston, 2007). Em 2003, as tendências da ocorrência dos urólitos em felinos começaram a modificar-se novamente. No Centro de Urólitos de Minnesota (CUM), a ocorrência de urólitos de estruvita voltaram a aumentar e em 2005, o número de urólitos de estruvitas foi observado na mesma proporção do de oxalato de cálcio (Osborne, Lulich, Forrester \& Albasan, 2009).

\section{Tratamento e prevenção}

Se tratando dos urólitos de estruvita, o objetivo do tratamento clínico é a modificação da dieta, dissolução do urólito e prevenção de recidivas, e depende da causa base da formação do urólito por estruvita (Del Angel-Caraza, 2010).

Em gatos com estruvita estérea, uma dieta acidificante com quantidades reduzidas de magnésio é indicada (Assis \& Taffarel, 2018). O uso da dieta terapêutica tende a dissolver os urólitos em torno de duas a seis semanas (Osborne et al, 2009). Deve-se considerar que dietas acidificantes podem predispor a formação de cristalúria por oxalato de cálcio (Koehler, Osborne, Buettner, Lulich \& Behnke, 2009), portanto é indispensável o monitoramento do paciente nesse período (Ulrich, Osborne, Cokley \& Lulich, 2009).

Também é indicado uma ingestão hídrica adequada para que haja diminuição da densidade urinária e micções com maior frequência, evitando que a urina fique muito concentrada (Assis \& Taffarel, 2018). O aumento do volume urinário pode ser feito através do uso de dietas úmidas específicas para gatos ou adicionando água no alimento seco oferecido (Rosa, 2011), contato que o gato se adapte à essas estratégias.

Nos casos de infecção, é importante a associação de uma dieta adequada e o uso de agentes antimicrobianos, com base em cultura bacteriológica e antibiograma (Bartges \& Callen, 2015; Lulich et al., 2016).

O tratamento cirúrgico só é indicado quando a dissolução dos cálculos não é possível (Filho, Prado, Ribeiro \& Fortes, 2013), em casos de obstrução ou onde seu tamanho impossibilita sua eliminação (Lulich, Osborne, Bartges \& Lekcharoensuk, 2015). E é de extrema importância, mesmo após o manejo terapêurico, o acompanhamento desses pacientes considerados predispostos para diagnóstico precoce, bom prognóstico e qualidade de vida (Barwaldt, Bierhals, de Lima, Silva \& Ferraz, 2021).

Já nos casos de urólitos de oxalato de cálcio, a causa da formação não é completamente elucidada, e nenhum tratamento se mostrou completamente efetivo (Bartges \& Callens, 2015). Como o oxalato de cálcio não é passível de dissolução, o objetivo do tratamento clínico é principalmente prevenir a sua formação corrigindo ou minimizando os fatores metabólicos conhecidos por aumentar o risco de formação desse tipo de urólito. Na confirmação do diagnóstico, o tratamento cirúrgico com remoção dos cálculos ou hidropropulsão são os métodos mais indicados (Assis \& Taffarel, 2018).

A recidiva de cálculos de oxalato de cálcio em gatos é um problema potencial (Bartges, 2016), o que reforça a importância de protocolos terapêuticos destinados a diminuir a recorrência de urólitos após a remoção, para isso, é essencial 
que haja o aumento da ingestão hídrica, redução de alimentos ricos em oxalato, utilização de rações terapêuticas para o controle da acidiúria mantendo o pH entre 7,0 e 7,5 como medidas preventivas (Little, 2016). Para promover a reabsorção de cálcio, suplementação com vitamina B6 e hidroclorotiazida pode ser utilizada (Assis \& Taffarel, 2018).

\section{Diagnóstico}

Os sinais clínicos dos felinos acometidos por urolitíases variam conforme a localização, número e tamanho dos cálculos, independente da composição (Houston, Vanstone, Moore \& Weese, 2016). O diagnóstico é feito com base no histórico do animal, anamnese, exames físicos e laboratoriais, como a urinálise, urocultura e bioquímico, e exames de imagem como ultrassonografia e radiografia, sendo de grande importância no diagnóstico, pois permitem a visualização dos cálculos (Vargas, De Campos, Blankenheim, \& Gomes, 2019). O diagnóstico definitivo de qualquer urólito é realizado mediante análise química do mesmo (Bartges \& Callens, 2015).

A radiografia abdominal simples é uma ferramenta diagnóstica muito útil, sendo geralmente a primeira modalidade de diagnóstico por imagem utilizada para detecção de urólitos, devendo abranger desde o diafragma até a porção final da uretra (Bartges \& Callens, 2015).

Os urólitos de estruvita e oxalato de cálcio são facilmente visualizados na radiografia, pois eles são radiopacos (Lulich, Osborne, Bartges \& Lekcharoensuk, 2015). A radiodensidade destes urólitos pode mudar para radioluscentes quando avaliados pela radiografia com duplo contraste (Nelson \& Couto, 2015; Rick et al., 2017).

Como as urolitíases são cada vez mais observadas em gatos com doença renal crônica (Kyles et al, 2005), a radiografia abdominal é recomendada para todos os gatos com esta afecção diagnosticada (Ross, Osborne, Lekcharoensuk, Koehler \& Polzin, 2007).

Se o cálculo apresentar dimensões inferiores a $4 \mathrm{~mm}$ e não for radiopaco o suficiente, sua visualização pelo exame radiográfico simples poderá ser prejudicada (Lulich \& Osborne, 2009). Nesse caso, a cistografia de duplo contraste ou a ultrassonografia (US) é superior à radiografia (Lulich, Osborne \& Albasan, 2011).

A utilização da radiografia contrastada é indicada para gatos com quadro clínicos recorrentes ou prolongadas (Hostutler, Chew \& DiBartola 2005), sendo indicados para evidenciar cálculos radiotransparentes, neoplasias, estenoses ou obstrução uretral (Reche \& Camozzi, 2015).

A ultrassonografia tem boa acurácia no diagnóstico das urolitíases, podendo detectar urólitos que não são passíveis de identificação na radiografia (Grauer, 2015) entretanto, não fornece informações suficientes quanto às características dos cálculos (tamanho, formato, radiopacidade e número) (Lulich, Osborne \& Albasan, 2011). Também tem boa aplicabilidade na diferenciação de dilatações pélvicas, verificação da integridade do trato urinário e detecção da presença de tampões na vesícula urinária (Galvão, Ondani, Frazílio \& Ferreira, 2010). A sensibilidade da ultrassonografia para a detecção de cálculos ureterais é de $77 \%$, o que pode chegar a $90 \%$ usando uma combinação de US e radiografia (Kyles et al., 2005).

O US indica a localização exata do urólito e também avalia o grau de obstrução, sendo principalmente indicada para a visualização da uretra proximal, possível de ser avaliada apenas por meio da ultrassonografia (Magalhães, 2013).

\section{Conclusão}

Nas produções analisadas, verificou-se que a obstrução uretral é uma das afecções mais comuns na medicina felina, onde as urolitíases são a principal causa. Os sinais clínicos apresentados pelos animais obstruídos são semelhantes à diversas outras afecções que acometem o trato urinário inferior desses animais. É considerado uma emergência clínica e pode resultar no óbito do paciente se não diagnosticada e tratada de forma adequada. 
A nutrição tem um papel importante na prevenção e resolução das urolitíases e suas recidivas, e o aumento da ingestão hídrica deve ser estimulada.

O acompanhamento clínico dos animais com dietas terapêuticas deve ser realizado, assim como animais com comorbidades que favorecem o desenvolvimento de urólitos, utilizando-se de exames complementares como o hemograma, perfil bioquímico, urinálise, radiografia e a ultrassonografia.

A urolitíase não deve ser considerada uma enfermidade com causa pontual, e sim consequência de múltiplas alterações sistêmicas em interação.

Assim, essa revisão consiste em um conjunto de estudos com bases sólidas e artigos atualizados sobre o tema, podendo servir de impulso para nortear outras pesquisas no assunto, visto a necessidade de elucidar melhor alguns aspectos na etiopatogenia das urolitíases e seus tratamentos.

\section{Referências}

Assis, M., \& Taffarel, M. (2018). Doença do trato urinário inferior dos felinos: abordagem sobre cistite idiopática e urolitíase em gatos. Enciclopedia Biosfera, 15(27).

Balaji, K. C., \& Menon, M. (1997). Mechanism of stone formation. Urologic Clinics of North America, 24(1), 1-11.

Bartges, J. W. (2016). Feline calcium oxalate urolithiasis: risk factors and rational treatment approaches. Journal of Feline medicine and Surgery, 18(9), 712722 .

Bartges, J. W., \& Kirk, C. A. (2006). Nutrition and lower urinary tract disease in cats. Veterinary Clinics: Small Animal Practice, 36(6), 1361-1376.

Bartges, J. W., Osborne, C. A., Lulich, J. P., Kirk, C., Allen, T. A., \& Brown, C. (1999). Methods for evaluating treatment of uroliths. Veterinary Clinics of North America: Small Animal Practice, 29(1), 45-57.

Bartges, J. W., \& Callens, A. J. (2015). Urolithiasis. Veterinary Clinics: Small Animal Practice, 45(4), 747-768.

Barwaldt, E. T., Bierhals, E. S., de Lima, C. M., da Silva, A. B., \& Ferraz, A. (2021). Urolitíase em filhote canino: Relato de caso. Research, Society and Development, 10(1).

Cooper, E. S. (2015). Controversies in the management of feline urethral obstruction. Journal of Veterinary Emergency and Critical Care, $25(1)$, $130-137$.

Daudon, M., Letavernier, E., Frochot, V., Haymann, J. P., Bazin, D., \& Jungers, P. (2016). Respective influence of calcium and oxalate urine concentration on the formation of calcium oxalate monohydrate or dihydrate crystals. Comptes Rendus Chimie, 19(11-12), 1504-1513.

Del Angel-Caraza, J., Diez-Prieto, I., Pérez-García, C. C., \& García-Rodríguez, M. B. (2010). Composition of lower urinary tract stones in canines in Mexico City. Urological research, 38(3), 201-204.

Dulaney, D. R, Hopfensperger, M., Malinowski, R., Hauptman, J., \& Kruger, J. M. (2017). Quantification of urine elimination behaviors in cats with a video recording system. Journal of veterinary internal medicine, 31(2), 486-491.

Ettinger, S. J., \& Feldman, E. C. (2004). Tratado de medicina interna veterinária: doenças do cão e do gato. afecções do estômago. 5ed. Rio de Janeiro: Guanabara Koogan, 1583-1614.

Ferreira, G. S. (2013). Características epidemiológicas, clínicas e laboratoriais de gatos com sinais de trato urinário inferior. Archives Of Veterinary Science, $19(4), 42-50$

Filho, E. S, Prado, T., Ribeiro, R., \& Fortes, R. (2013). Urolitíase canina. Enciclopédia Biosfera, 9(17).

Galvão, A. L. B., Ondani, A. C., Frazílio, F. O., \& Ferreira, G. S. (2010). Obstrução uretral em gatos machos-revisão literária. Acta Veterinaria Brasilica, 4(1), 1-6.

Giovaninni, L. H., \& Piai, V. D. S. (2010). O uso da acupuntura no auxílio à terapia da doença idiopática do trato urinário inferior dos felinos. Ciência Rural, 40(3), 712-717.

Grauer, G. F. (2015). Feline struvite \& calcium oxalate urolithiasis. Todays Vet Pract, 5(5), 14-20.

Guerra, M. G. (2018). Urolitíase no trato urinário inferior em cães: revisão de literatura. Repositório Institucional Unisa, Santo Amaro, 25.

Hostutler, R. A., Chew, D. J., \& DiBartola, S. P. (2005). Recent concepts in feline lower urinary tract disease. Veterinary Clinics: Small Animal Practice, 35(1), 147-170.

Houston, D. M., Vanstone, N. P., Moore, A. E., Weese, H. E., \& Weese, J. S. (2016). Evaluation of 21426 feline bladder urolith submissions to the Canadian Veterinary Urolith Centre (1998-2014). The Canadian Veterinary Journal, 57(2), 196. 
Houston, D. M., Moore, A. E., Favrin, M. G., \& Hoff, B. (2003). Feline urethral plugs and bladder uroliths: a review of 5484 submissions 1998-2003. The Canadian Veterinary Journal, 44(12), 974.

Houston, D. (2007). Epidemiología de la urolitiasis felina. Veterinary focus, 17, 4-9.

Koehler, L. A., Osborne, C. A., Buettner, M. T., Lulich, J. P., \& Behnke, R. (2009). Canine uroliths: frequently asked questions and their answers. Veterinary Clinics of North America: Small Animal Practice, 39(1), 161-181.

Kyles, A. E., Hardie, E. M., Wooden, B. G., Adin, C. A., Stone, E. A., Gregory, C. R., ... \& Ling, G. V. (2005). Clinical, clinicopathologic, radiographic, and ultrasonographic abnormalities in cats with ureteral calculi: 163 cases (1984-2002). Journal of the American Veterinary Medical Association, 226(6), 932-936.

Lekcharoensuk, C., Osborne, C. A., \& Lulich, J. P. (2001). Epidemiologic study of risk factors for lower urinary tract diseases in cats. Journal of the American Veterinary Medical Association, 218(9), 1429-1435.

Lekcharoensuk, C., Lulich, J. P., Osborne, C. A., Koehler, L. A., Urlich, L. K., Carpenter, K. A., \& Swanson, L. L. (2000). Association between patientrelated factors and risk of calcium oxalate and magnesium ammonium phosphate urolithiasis in cats. Journal of the American Veterinary Medical Association, 217(4), 520-525.

Lima, E. R., Reis, J. C., Menezes, M.M., Santos, F.L., Pereira, M. F., Almeida, E.L., Teixeira, M. N., \& Silva, M. G. V. (2008). Aspectos anatomopatológicos em gatos domésticos com doença do trato urinário inferior. Medicina Veterinária (UFRPE), 2(4), 17-26.

Little, S. E. (2016). Trato urinário inferior. In O Gato - Medicina Interna (pp. 944 - 975). Rio De Janeiro: Rocca.

Little, S.E. (2012). Lower urinary tract. In The Cat Clinical Medicine And Management. (pp. 935-1013). Ottawa: Elselvier Saunders

Lulich, J. P., Osborne, C. A., \& Albasan, H. (2011). Canine and feline urolithiasis: diagnosis, treatment, and prevention. In Nephrology and urology of small animals (pp. 687-706). Wiley.

Lulich, J. P., \& Osborne, C. A. (2009). Changing paradigms in the diagnosis of urolithiasis. Veterinary Clinics of North America: Small Animal Practice, 39(1), 79-91.

Lulich, J. P., Berent, A. C., Adams, L. G., Westropp, J. L., Bartges, J. W., \& Osborne, C. A. (2016). ACVIM small animal consensus recommendations on the treatment and prevention of uroliths in dogs and cats. Journal of veterinary internal medicine, 30(5), 1564-1574.

Macphail, C. M. (2014). Cirurgia da bexiga e da uretra. FOSSUM, TW Cirurgia de pequenos animais, 4, $2162-2170$.

Magalhães F. A (2013). Urolitíase Em Cães. Monografia, Lume, Universidade Federal Do Rio Grande Do Sul, Porto Alegre, Brasil.

Marquez, G. A., Klausner, J. S., \& Osborne, C. A. (1995). Calcium oxalate urolithiasis in a cat with a functional parathyroid adenocarcinoma. Journal of the American Veterinary Medical Association, 206(6), 817-819.

Martins, G. S., de Cassia Martini, A., Meirelle, Y. S., Dutra, V., Nespóli, P. E. B., Mendonça, A. J., \& Sousa, V. R. F. (2013). Avaliação clínica, laboratorial e ultrassonográfica de felinos com doença do trato urinário inferior. Semina: Ciências Agrárias, 34(5), 2349-2355.

Mayer-Roenne, B., Goldstein, R. E., \& Erb, H. N. (2007). Urinary tract infections in cats with hyperthyroidism, diabetes mellitus and chronic kidney disease. Journal of feline medicine and surgery, 9(2), 124-132.

Midkiff, A. M., Chew, D. J., Randolph, J. F., Center, S. A., \& DiBartola, S. P. (2000). Idiopathic hypercalcemia in cats. Journal of Veterinary Internal Medicine, 14(6), 619-626.

Moore, A. (2007). Quantitative analysis of urinary calculi in dogs and cats. Vet Focus, 17, 22-27.

Nelson, R., \& Couto, C. G. (2015). Medicina interna de pequenos animais. Elsevier Brasil.

Nelson, R.W.; Couto, C. G. (2001). Medicina interna de pequenos animais. Guanabara Kogan.

Osborne, C. A., Lulich, J. P., Bartges, J. W., Unger, L. K., Thumchai, R., Koehler, L. A. \& Felice, L. J. (1995). Canine and feline urolithiasis: relationship of etiopathogenesis to treatment and prevention. Canine and feline nephrology and urology, 37(12), 798-888.

Osborne, C. A., Lulich, J. P., Forrester, D., \& Albasan, H. (2009). Paradigm changes in the role of nutrition for the management of canine and feline urolithiasis. Veterinary Clinics of North America: Small Animal Practice, 39(1), 127-141.

Osborne, C. A., Lulich, J. P., Kruger, J. M., Ulrich, L. K., \& Koehler, L. A. (2009). Analysis of 451,891 canine uroliths, feline uroliths, and feline urethral plugs from 1981 to 2007: perspectives from the Minnesota Urolith Center. Veterinary Clinics of North America: Small Animal Practice, $39(1), 183-197$.

Oyafuso, M. K., Kogika, M. M., Waki, M. F., Prosser, C. S., Cavalcante, C. Z., \& Wirthl, V. A. B. F. (2010). Urolitíase em cães: avaliação quantitativa da composição mineral de 156 urólitos. Ciência Rural, 40, 102-108.

Palm, C. A., \& Westropp, J. L. (2011). Cats and calcium oxalate: strategies for managing lower and upper tract stone disease. Journal of feline medicine and surgery, 13(9), 651-660.

Pimenta, M. M., Reche-Júnior, A., Freitas, M. F., Kogika, M. M., \& Hagiwara, M. K. (2014). Estudo da ocorrência de litíase renal e ureteral em gatos com doença renal crônica. Pesquisa Veterinária Brasileira, 34, 555-561.

Reche Junior, A., \& Camozzi, R. B. (2015). Doença do trato urinário inferior dos felinos-Cistite intersticial. Jerico MM, Andrade neto JP, Kogika MM. Tratado de medicina interna de cães e gatos. la ed. São Paulo: Roca, 2, 1483-92. 
Research, Society and Development, v. 10, n. 8, e51910817094, 2021

(CC BY 4.0) | ISSN 2525-3409 | DOI: http://dx.doi.org/10.33448/rsd-v10i8.17094

Rick, G. W., Conrad, M. L. H., de Vargas, R. M., Machado, R. Z., Lang, P. C., Serafini, G. M. C., \& Bones, V. C. (2017). Urolitíase em cães e gatos. PUBVET, 11, 646-743.

Robertson, E. (2014). Feline cystitis: a case presenting with LUTS in a young female cat. Companion Animal, 19(6), $284-287$.

Rosa, L. S. (2011). Doença do trato urinário inferior felino. PUBVET, 5, 1258-1263.

Ross, S. J., Osborne, C. A., Lekcharoensuk, C., Koehler, L. A., \& Polzin, D. J. (2007). A case-control study of the effects of nephrolithiasis in cats with chronic kidney disease. Journal of the American Veterinary Medical Association, 230(12), 1854-1859.

Stevenson, A. E. (2002). The incidence of urolithiasis in cats and dogs, and the influence of diet in formation and prevention of recurrence (Doctoral dissertation, University of London, University College London (United Kingdom)).

Ulrich, L. K., Osborne, C. A., Cokley, A., \& Lulich, J. P. (2009). Changing paradigms in the frequency and management of canine compound uroliths. Veterinary Clinics of North America: Small Animal Practice, 39(1), 41-53.

Vargas, M. E. B., De Campos, M., Blankenheim, T. M., \& Gomes, D. E. (2019). Urolitíase-revisão de literatura. Revista científica, 1(1). 